\title{
L'incidence de l'hypotension post-intubation endotrachéale chez des patients en salle de réanimation: impact des définitions
}

\author{
Marie-Claire Lévesque, $\mathrm{MD}^{*^{+}}$; Natalie Le Sage, $\mathrm{MD}, \mathrm{PhD}^{*^{+}}$; Simon Berthelot, $\mathrm{MD}, \mathrm{MSc}^{* \dagger}$; \\ Valérie Boucher, BA*; Éric Mercier, $\mathrm{MD}^{*{ }^{\dagger}}$; Marcel Émond, MD, $\mathrm{MSc}^{*{ }^{\dagger \ddagger}}$
}

\section{ABSTRACT}

Objectif: Notre objectif primaire est de mesurer l'incidence d'HPI selon quatre définitions différentes retrouvées dans la littérature. Notre principal objectif secondaire est d'évaluer l'impact de la présence d'instabilité hémodynamique avant I'intubation sur l'incidence d'HPI. Le deuxième objectif secondaire consiste à déterminer l'incidence de I'HPI en fonction de l'intervalle de temps durant lequel la première hypotension survient.

Méthode: Une cohorte prospective a été constituée par les patients intubés en salle de réanimation à l'hôpital de I'Enfant-Jésus entre le 28/06/2011 et le 12/07/2012. L'HPI était globalement définie comme $\geq 1$ mesure de tension artérielle systolique $<90 \mathrm{mmHg}$ suivant l'intubation. Les différentes définitions étudiées faisaient varier le temps de mesure de la tension artérielle (TA) après l'intubation, soit 1) jusqu'à 5 minutes, 2) jusqu'à 15 minutes, 3) jusqu'à 30 minutes et 4) en tout temps lors du séjour en salle de réanimation.

Résultat: Au cours de la période à l'étude, 155 patients ont été intubés sur place dont 81 patients qui répondaient aux critères d'inclusion. L'incidence de I'HPI pour chaque définition est respectivement de $9.9 \%, 18.5 \%, 24.7 \%$ et $28.4 \%$. La comparaison entre chacune de ces incidences révèle une différence statistiquement significative $(p<0.05)$, à l'exception des deux dernières. L'incidence cumulative d'HPI à tout moment suivant I'intubation chez les patients présentant une hypotension préintubation est de $62.5 \%$ (IC 95\% 28.5-87.5) en opposition aux patients hémodynamiquement stables en pré-intubation qui présentaient une incidence d'HPI de 24.7\% (IC 95\% 16.1-35.8). Conclusion : L'hypotension post-intubation est un effet indésirable fréquent chez les patients au département d'urgence et son incidence varie de façon significative en fonction de la définition temporelle utilisée au sein d'une même cohorte de patients.

Keywords: intubation, hypotension, soins d'urgence

\section{INTRODUCTION}

L'intubation endotrachéale (IET) est une technique fréquemment utilisée dans les urgences en salle de réanimation et qui s'avère potentiellement salvatrice chez les patients en situation critique. ${ }^{1-3}$ En effet, les urgentologues sont les intervenants les plus souvent impliqués dans la reconnaissance de l'insuffisance respiratoire et l'initiation subséquente d'IET. ${ }^{4}$ L'IET est une importante technique qui devient de plus de plus un facteur significatif influençant le pronostic des patients qui y sont soumis. ${ }^{5}$ Cependant, il est rapporté que, lorsque comparé à des méthodes de ventilation non-invasives, l'IET est associée à davantage d'évènements indésirables. ${ }^{6-8}$ Ces effets indésirables peuvent être de nature mineure ou majeure, allant du trauma dentaire aux altérations hémodynamiques telles que la bradycardie, l'hypotension ou même l'arrêt cardio-respiratoire. Le décès est aussi un évènement redouté pouvant survenir. ${ }^{9,10}$ L'hypotension postintubation (HPI) est l'un des effets indésirables connus rapporté dans la littérature. ${ }^{11}$

Les différents facteurs de risque d'HPI reconnus sont un diagnostic de sepsis, la présence de maladie pulmonaire obstructive chronique (MPOC), ${ }^{11,12}$ une tension artérielle systolique (TAS) de moins de $140 \mathrm{mmHg}$ avant l'intubation, ${ }^{11}$ l'âge avancé et une instabilité hémodynamique pré-intubation. Notons que d'autres facteurs ont été mentionnés tels que le poids inférieur à $55 \mathrm{~kg}{ }^{11}$ un indice de choc (shock index) élevé avant l'intubation et la présence de maladie rénale ou d'un

Du ${ }^{*}$ Centre de recherche du CHU de Québec, Axe Santé des populations et pratiques optimales en santé, Québec, QC; ${ }^{\dagger}$ Département de médecine familiale et médecine d'urgence, Université Laval, Québec, OC; et le ${ }^{\ddagger}$ Centre d’Excellence du Vieillissement de Québec, CIUSSS de la Capitale Nationale, Québec, QC.

Correspondance à: Marcel Émond, CHU de Québec, Hôpital de l'Enfant-Jésus 1401, 18e rue (H-601), Québec, OC G1J 1Z4; Email : marcelemond1@ me.com 
diagnostic d'insuffisance respiratoire aiguë. ${ }^{13} \mathrm{La}$ physiopathologie de l'HPI reste hypothétique, mais plusieurs mécanismes ont été proposés. L'effet hypotenseur de certains agents d'induction, le tonus sympathique abaissé des patients en sepsis davantage diminué par l'effet hémodynamique de l'intubation ainsi que la ventilation mécanique qui diminue le retour veineux au ventricule droit sont divers mécanismes proposés d'HPI. ${ }^{14-17}$

Il est bien documenté que l'hypotension chez une population gravement malade est potentiellement associée à une mortalité et une morbidité importantes. ${ }^{18-20}$ Toutefois, des données conflictuelles existent en regard d'une association possible entre l'HPI et une mortalité augmentée. Jusqu’à présent, deux études ont exploré la possibilité d'une telle association : Green et al ont évalué s'il y avait une augmentation de la mortalité ainsi que de la durée de séjour hospitalier chez les patients atteints d'HPI à la salle d'urgence, mais aucune association significative entre ces variables n'a été montrée. ${ }^{12}$ Cette association a cependant été observée par Heffner et al la même année. ${ }^{13}$ Ils possédaient un échantillon plus large de 336 patients au DU (département d'urgence), grâce auquel ils ont pu identifier l'HPI comme un prédicteur significatif de mortalité intra hospitalière augmentée. Cependant, les définitions d'HPI utilisées étaient différentes entre ces deux études et pourraient expliquer ces résultats contradictoires.

Des données conflictuelles existent également sur l'incidence de l'HPI, ${ }^{7,12,21-24}$ certains auteurs suggérant qu'il s'agit d'une complication rare $^{25}$ et d'autres rapportant cet effet indésirable comme relativement commun. ${ }^{7,23,26,27}$ Son incidence varie effectivement de $0 \%$ à $44 \%$ si l'on considère les études disponibles sur le sujet. $^{7,21-24}$ Tant pour l'évaluation de son impact sur la mortalité que pour l'estimation de son incidence, la grande hétérogénéité des définitions d'HPI utilisées affectent la comparabilité des résultats et affaiblissent les conclusions cliniques potentielles. Une mesure de TAS (tension artérielle systolique) $\leq 90 \mathrm{mmHg}$ est fréquemment utilisée afin de conclure à la présence d'hypotension, mais cette définition ne fait pas l'unanimité. ${ }^{16,17,25,28-31}$ Certains auteurs ont en effet proposé qu'une diminution $\geq 20 \%$ de la TAS par rapport à la TAS de base, ${ }^{12,30}$ le besoin de vasopresseur ${ }^{12,30}$ ou encore une tension artérielle moyenne $\leq 65 \mathrm{mmHg}$, pourraient être des mesures alternatives afin d'évaluer la présence d'HPI. De plus, la durée pendant laquelle la TAS est mesurée après l'intubation est variable, allant de 5 à 60 minutes lorsque précisée dans les études. ${ }^{11-13,32}$ Nous émettons l'hypothèse que le paramètre de temps des définitions influence l'incidence d'HPI.

L'objectif principal de cette étude est d'évaluer l'impact du choix de la définition d'HPI sur son incidence. Le premier objectifs secondaire consiste à décrire l'incidence d'HPI en comparant les patients hypotendus (TAS $\leq 90 \mathrm{mmHg}$ ) aux patients considérés normotendus (TAS $>90 \mathrm{mmHg}$ ) avant l'intubation, et ce, selon six définitions. Le deuxième objectif secondaire consiste à déterminer l'incidence de l'HPI (définition TAS $\leq 90$ $\mathrm{mmHg}$ ) en fonction de l'intervalle de temps durant lequel la première hypotension est survenue.

\section{MÉTHODES}

\section{Devis de recherche et population}

Nous avons mené une étude de cohorte consécutive prospective au Centre Hospitalier Universitaire de l'Enfant-Jésus (CHU-HEJ) de Québec de juin 2011 à juillet 2012. Les patients inclus dans cette étude devaient être âgés de 16 ans ou plus ainsi qu'être intubés en salle de réanimation au département d'urgence lors d'une situation critique sans égard à la méthode d'IET utilisée (ex. laryngoscopie directe, vidéolaryngoscopie ou autres) à l'aide d'une intubation à séquence rapide utilisant la curarisation.

Les patients ayant été intubés avant leur arrivée à l'urgence, comme dans le cas d'un transfert inter hospitalier, étaient exclus. Il est à noter que le personnel pré hospitalier ne procède pas à l'IET dans notre province. Les autres critères d'exclusion étaient le besoin d'un accès aux voies aériennes de façon chirurgicale, un échec d'IET, l'absence de curare et, finalement, l'arrêt cardiorespiratoire avant l'IET. L'IET était défini comme le passage d'un tube endotrachéal à travers la glotte chez un patient afin de maintenir les voies respiratoires ouvertes. Dans notre centre, l'intubation est faite de routine par l'urgentologue sur place ou par un résident sous supervision. La pré-oxygénation est utilisée. L'utilisation du laryngoscope ou de video-laryngoscope est laissée à la discrétion de l'urgentologue.

\section{Cueillette des données}

Tous les patient étaient pris en charge à l'aide du système électronique de support de décision clinique (SEDC) ReaScribe + (Logibec inc) installé dans chaque salle de 
réanimation depuis juin 2011 qui permet un recueil standardisé ainsi qu'automatisé des signes vitaux des patients.

Les informations enregistrées par ReaScribe + agissaient à titre de dossier médical électronique pour l'épisode en salle de réanimation puisque l'ensemble des interventions médicales étaient inscrites par les infirmières via l'interface munie d'un écran tactile. Lorsqu'un patient avait plus d'un épisode d'intubation à son actif (ex. lors de différente visites à l'urgence), nous avons considéré uniquement le premier épisode d'intubation. La TA (tension artérielle) mesurée par brassard, était recueillie automatiquement par défaut à toutes les deux minutes, permettant de déterminer de façon fiable et systématique la présence ou l'absence d'HPI. Ces données étaient directement exportées vers le logiciel de recueil en temps réel. De plus, afin de valider les mesures automatiques de la plate-forme, la première mesure de TA était prise de façon manuelle par l'infirmière et inscrite aussi de façon manuelle à l'aide de l'interface tactile de la plate-forme. Les autres données recueillies via la plate-forme étaient : les caractéristiques démographiques, le moment de l'IET, la raison de consultation et la médication utilisée pour l'IET ainsi que les doses reçues.

\section{Définitions des issues}

Il n'existe pas de définition standard pour l'HPI. Suite à la révision de la littérature, un comité aviseur (MCL, ME, NL) a choisi une valeur d'HPI qui a l'avantage d'être souvent utilisée et facilement applicable par les cliniciens en situation d'urgence, c'est-à-dire la présence d'au moins une mesure de TAS de $90 \mathrm{mmHg}$ ou moins. ${ }^{11-13,16,17,25,28-33}$ Les définitions détaillées afin de constituer nos sous-groupes dans le cadre de notre objectif primaire étaient aussi prédéfinies (Tableau 1). La première définition testée tenait compte des mesures de TAS jusqu'à 5 minutes post IET. La deuxième définition testée tenait compte des mesures de TAS

\begin{tabular}{|l|l|}
\hline Tableau 1. Légende des définitions \\
\hline Définition & Temps de mesure de la TA post-intubation \\
\hline 1 & TAS $\leq 90 \mathrm{~mm} \mathrm{Hg}$ Jusqu'à 5 minutes \\
2 & TAS $\leq 90 \mathrm{~mm} \mathrm{Hg}$ Jusqu'à 15 minutes \\
3 & TAS $\leq 90 \mathrm{~mm} \mathrm{Hg}$ Jusqu'à 30 minutes \\
4 & TAS $\leq 90 \mathrm{~mm} \mathrm{Hg}$ en tout temps \\
5 & Utilisation de vasopresseurs en tout temps \\
6 & TAM $\leq 65 \mathrm{~mm} \mathrm{Hg}$ en tout temps \\
\hline
\end{tabular}

jusqu'à 15 minutes. La troisième définition testée tenait compte des mesures de TAS jusqu'à 30 minutes. La quatrième et dernière définition testée tenait compte de toutes les mesures de TAS mesurées après l'intubation lors du séjour en salle de réanimation. Deux autres définitions d'HPI ne tenant pas compte du moment de mesure de la TA ont été explorées : la TAM (tension artérielle moyenne) $\leq 65 \mathrm{mmHg}$ ainsi que l'utilisation de vasopresseurs. De la même façon, l'hypotension pré-intubation était définie comme la présence d'au moins une mesure de TAS de $90 \mathrm{mmHg}$ ou moins. Les mesures de tension jusqu'à 15 minutes avant l'intubation étaient prises en considération pour tous les patients. Les intervalles de temps du deuxième objectif secondaire étaient : 0 à 5 minutes, 6-15 minutes, 16 à 30 minutes et, finalement, 31 minutes et plus.

Les résultats sont présentés sous forme de proportions avec des intervalles de confiance fixés à $95 \%$. Un modèle d'équations d'estimation généralisées (GEE) a été utilisé. La comparaison des incidences obtenues a été effectuée à l'aide d'un test du $\chi^{2}$ de Wald. Un calcul de puissance à posteriori en regard de notre objectif principal a été effectué à l'aide d'un test de McNemar (Annexe 1).

Toutes les analyses ont été effectuées à l'aide du logiciel $S A S$ 9.4, Canada. L'étude a été approuvée par le comité d'éthique du centre hospitalier du CHU de Québec.

\section{RÉSULTATS}

\section{Données démographiques}

Au cours de la période à l'étude, 2275 patients ont été évalués en salle de réanimation de l'Enfant-Jésus. Au total, 219 patients intubés ont été recensés et de ce nombre, 155 patients ont été intubés au DU. Finalement, de ces 155 patients, 81 patients répondaient aux critères d'inclusion et 74 patients ont été exclus, le plus souvent pour des raisons techniques reliées au degré de confort variable du personnel vis-à-vis l'utilisation de la plateforme durant la période de son implantation et l'absence de curare (Annexe 2: $\mathrm{n}=5$ puisque $<16$ ans, $\mathrm{n}=30$ pour TAS manquante ou seulement des valeurs avant ou après intubation de TAS, $\mathrm{n}=5$ erreur d'enregistrement, $\mathrm{n}=34$ pour l'absence de bloquant neuro-musculaire). Les caractéristiques démographiques et cliniques des patients inclus sont rapportées dans le Tableau 2. L'âge moyen était de 54 ans (e.t. +/-19) et 64\% étaient des hommes. La raison de consultation la plus fréquente était une altération de l'état de conscience (53.1\%), suivi par un 


\begin{tabular}{|c|c|c|c|}
\hline Cohorte & Tous, $\mathrm{n}=81$ & $\mathrm{HPI}+, \mathrm{n}=23$ & $\mathrm{HPI}-, \mathrm{n}=58$ \\
\hline \multicolumn{4}{|l|}{ Caractéristiques } \\
\hline Âge (années), moyenne \pm e.t.* & $54 \pm 19$ & $59 \pm 18$ & $52 \pm 19$ \\
\hline Âge (années), médiane $(I I Q)^{\dagger}$ & $56(39-69)$ & $63(49-74)$ & $53(36-64)$ \\
\hline Sexe (masculin), n (\%) & $51(63.8)$ & $14(60.9)$ & $37(64.9)$ \\
\hline Temps en salle de réanimation (min) médiane (IIQ) & $51(40-70)$ & $61(45-103)$ & $49(39-64)$ \\
\hline Mesure de TAS disponibles pré-IET ${ }^{\ddagger}$ et post-IET (médiane (IIQ)) & $14(9-19)$ & $18(15-24)$ & $13(8-18)$ \\
\hline Mesure de TAS disponibles post-IET (médiane (IIQ)) & $10(7-15)$ & $14(10-22)$ & $8(6-13)$ \\
\hline \multicolumn{4}{|l|}{ Raisons de consultation, $\mathrm{n}(\%)$} \\
\hline Traumatisme & $24(29.6)$ & $5(21.7)$ & $19(32.8)$ \\
\hline Douleur rétro-sternale & $2(2.5)$ & $4(4.4)$ & $1(1.7)$ \\
\hline Dyspnée & $7(8.6)$ & $5(21.7)$ & $2(3.5)$ \\
\hline Altération de l'état de conscience & $43(53.1)$ & $10(43.5)$ & $33(56.9)$ \\
\hline Autres & $5(6.2)$ & $2(8.7)$ & $3(5.2)$ \\
\hline \multicolumn{4}{|l|}{ Médication utilisée pour l'intubation, n $(\%)^{\S}$} \\
\hline Fentanyl & $30(37.0)$ & $11(47.8)$ & $19(32.8)$ \\
\hline Étomidate & $47(58.0)$ & $11(47.8)$ & $36(62.1)$ \\
\hline Ketamine & $3(3.7)$ & $3(13.0)$ & $0(0.0)$ \\
\hline Propofol & $43(53.1)$ & $8(34.8)$ & $35(60.3)$ \\
\hline Midazolam & $20(24.7)$ & $8(34.8)$ & $12(20.7)$ \\
\hline Vasopresseur & $4(4.9)$ & $4(17.4)$ & $0(0.0)$ \\
\hline \multicolumn{4}{|c|}{$\begin{array}{l}\text { *e.t. (écart-type) } \\
\text { IIIQ (Interquartile); } \\
\text { fIET (intubation endotrachéale) } \\
\text { sLes catégories ne sont pas mutuellement exclusives, plus d'une médication ayant parfois été administrée au même patient. }\end{array}$} \\
\hline
\end{tabular}

traumatisme (29.6\%). L'agent d'induction le plus utilisé était l'etomidate $(58.0 \%)$, suivi par le propofol (53.1\%). Une cote de triage 1 (immédiat) selon l'échelle canadienne de triage (CTAS) (34) a été attribuée à 58 (74.4\%) des 81 patients alors que $21.8 \%$ des patients ont reçus une priorité de triage 2 (très urgent), $1.3 \%$ priorité 3 (urgent) et $2.6 \%$ priorité $\mathrm{P} 4$ (moins urgent). La médiane du nombre total (pré et post IET) de TA recueillies par patient était de 14 (IIQ (interquartile) : 9-19). L'intubation en raison d'un traumatisme ou d'une altération de l'état de conscience était plus fréquente chez les patients hémodynamiquement stables que chez les patients atteints d'HPI. Les patients développant de l'HPI ont davantage reçu du fentanyl et du midazolam lors de l'intubation à séquence rapide en comparaison avec les patients ne développant pas d'HPI, chez qui on observe l'utilisation plus marquée de propofol et/ou d'etomidate.

\section{Paramètres hémodynamiques}

Les paramètres hémodynamiques des patients, tels que la TAS moyenne, la TAD (tension artérielle diastolique) moyenne, la saturation moyenne et la fréquence cardiaque moyenne, sont présentés en fonction du temps écoulé

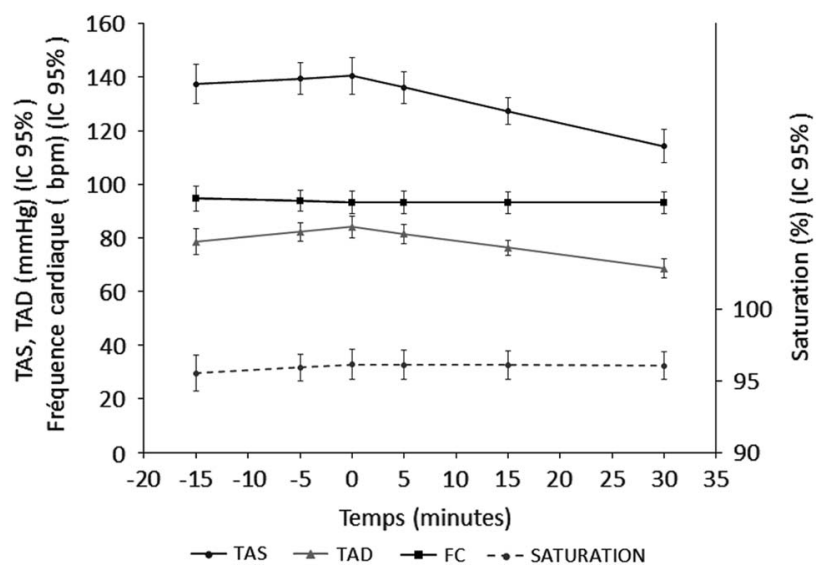

Figure 1. Distribution des paramètres hémodynamiques TAS : tension artérielle systolique; TAD : tension artérielle diastolique; FC : fréquence cardiaque.

depuis l'intubation (Figure 1). La période des 15 minutes précédant l'intubation a été inclue. Nous remarquons une diminution de la TAS moyenne et de la TAD moyenne durant la période post-intubation, alors que les autres paramètres sont stables.

De plus, les mêmes paramètres sont présentés d'une part chez les patients atteints d'HPI et, d'autre part, chez 
les patients hémodynamiquement stables (Figure 2). On note que la TAS moyenne, la TAD moyenne et la saturation moyenne sont plus basses chez les patients atteints d'HPI, et ce, peu importe le temps écoulé depuis l'intubation. De plus, la TAS et la TAD moyenne chez le groupe atteint d'HPI tendent à diminuer dans le temps. Cette descente s'amorce dès la période pré-intubation. Chez les patients non-atteints d'HPI, une tendance à la baisse est aussi notée, mais uniquement durant la période post-intubation. En effet, une légère augmentation de la tension artérielle est observée en pré intubation chez ce groupe dépourvu d'HPI.

\section{Incidences d'hypotension post-intubation}

L'HPI à tout moment a été observée chez 23 (28.4\%; IC $95 \%$ 20.1-40.1) des 81 patients à tout moment suivant l'intubation (Tableau 3) (définition \#4). L'incidence d'HPI augmente de façon graduelle lorsque le temps de

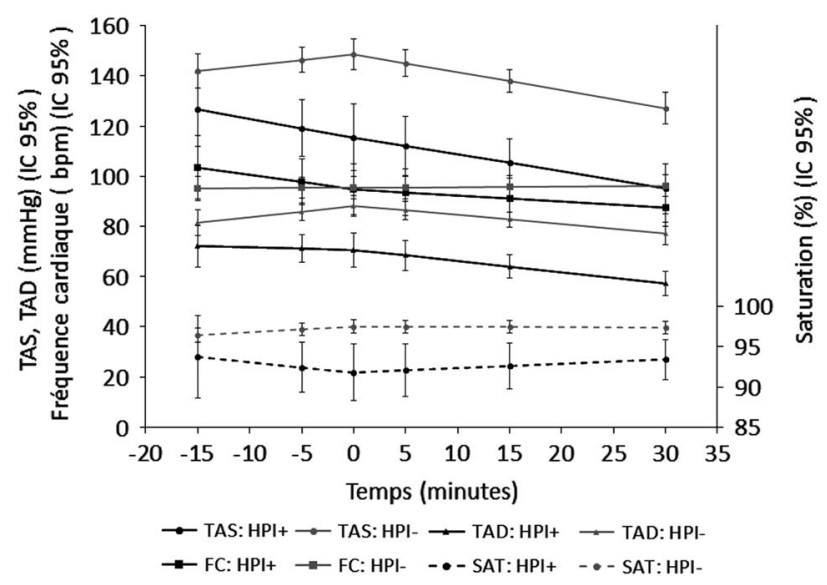

Figure 2. Distribution des paramètres hémodynamiques : en fonction de la présence d'HPI

TAS : tension artérielle systolique; TAD : tension artérielle diastolique; FC : fréquence cardiaque. mesure de TA augmente : l'incidence d'HPI était de $9.9 \%, 18.5 \%$ et $24.7 \%$ respectivement pour les définitions \#1, \#2 et \#3. Lors d'une comparaison entre les proportions d'incidences observées (définitions \#1 à 4), nous observons des différences statistiquement significatives entre les définitions $(p<0.05)$ pour chacune des comparaisons (Tableau 4), à l'exception de la comparaison entre les définitions 3 et 4 . L'incidence d'HPI déterminée par la présence de vasopresseurs (définition \#5) et par une TAM abaissée (définition \#6) est respectivement de 4.9\% et $28.4 \%$. Le temps médian de survenue de la première HPI était de13 minutes (IIQ 2.5-18.0).

$\mathrm{Au}$ total, l'incidence cumulative d'HPI à tout moment suivant l'intubation chez les patients présentant une hypotension pré-intubation était de $62.5 \%$ (IC 95\%28.5-87.5) (Tableau 5). Par opposition, l'incidence cumulative d'HPI à tout moment suivant l'intubation chez les patients ne présentant pas d'hypotension pré-intubation était de 24.7\% (IC 95\% 16.1-35.8). Quelle que soit la définition utilisée, l'incidence d'HPI est toujours significativement plus élevée en présence d'hypotension pré-intubation (Tableau 5).

L'incidence d'HPI (TAS $\leq 90 \mathrm{~mm} \mathrm{Hg}$ ) où survient la première hypotension en fonction des intervalles de temps 0 à 5 minutes, 6 à 15 minutes et 16 à 30 minutes est respectivement de $9.9 \%, 8.6 \%$ et $6.2 \%$. L'incidence

\begin{tabular}{|c|c|c|c|}
\hline Définitions & Comparaison & Valeur $Z$ & Valeur $p$ \\
\hline 1 vs 2 & $0-5 \min$ vs $0-15$ min & 2.60 & 0.0092 \\
\hline 1 vs 3 & $0-5$ min vs $0-30 \mathrm{~min}$ & 3.35 & 0.0008 \\
\hline 2 vs 3 & $0-15$ min vs $0-30 \mathrm{~min}$ & 2.23 & 0.0259 \\
\hline 1 vs 4 & $0-5$ min vs à tout moment & 3.70 & 0.0002 \\
\hline 2 vs 4 & $0-15$ min vs $S$ à tout moment & 2.81 & 0.0050 \\
\hline 3 vs 4 & 0-30 min vs à tout moment & 1.73 & 0.0835 \\
\hline
\end{tabular}

\begin{tabular}{|c|c|c|}
\hline Définition & Intervalle mesure de tension artérielle post-intubation & Incidence cumulative HPI (\%; IC* 95\%) \\
\hline 1 & TAS $\leq 90 \mathrm{~mm} \mathrm{Hg}: 0-5$ minutes & $8 / 81(9.9 ; 5.1-19.1)$ \\
\hline 2 & TAS $\leq 90 \mathrm{~mm} \mathrm{Hg}: 0-15$ minutes & $15 / 81(18.5 ; 11.7-29.2)$ \\
\hline 3 & TAS $\leq 90 \mathrm{~mm} \mathrm{Hg}: 0-30$ minutes & $20 / 81(24.7 ; 16.9-36.1)$ \\
\hline 4 & TAS $\leq 90 \mathrm{~mm} \mathrm{Hg}:$ À tout moment $^{\dagger}$ suivant l'intubation ( $\geq 0$ minutes) & $23 / 81(28.4 ; 20.1-40.1)$ \\
\hline 5 & Besoin de vasopresseurs & $4 / 81(4.9 ; 1.9-12.0)$ \\
\hline 6 & $\mathrm{TAM} \leq 65 \mathrm{mmHg}$ & $23 / 81(28.4 ; 19.7-39.0)$ \\
\hline
\end{tabular}




\begin{tabular}{|c|c|c|c|c|}
\hline \multirow[b]{2}{*}{ Définition } & \multirow[b]{2}{*}{$\begin{array}{l}\text { Intervalle de mesure de tension artérielle } \\
\text { post-intubation }\end{array}$} & \multicolumn{3}{|c|}{ Incidence cumulative d'HPI N (\%; IC 95\%) } \\
\hline & & $\begin{array}{l}\text { Hypotension pré-intubation } \\
\text { présente } \mathrm{N}=8^{*}\end{array}$ & $\begin{array}{l}\text { Hypotension pré-intubation } \\
\text { absente } \mathrm{N}=73\end{array}$ & Valeur $\mathrm{p}$ \\
\hline 1 & $0-5 \min$ & $4(50.0 ; 20.0-80.0)$ & $4(5.5 ; 2.1-13.7)$ & 0.0011 \\
\hline 2 & $0-15 \min$ & $5(62.5 ; 28.5-87.5)$ & $10(13.7 ; 7.5-23.6)$ & 0.0035 \\
\hline 3 & $0-30 \mathrm{~min}$ & $5(62.5 ; 28.5-87.5)$ & $15(20.6 ; 12.8-31.3)$ & 0.0177 \\
\hline 4 & $\grave{A}$ tout moment suivant l'intubation ( $\geq 0 \mathrm{~min}$ ) & $5(62.5 ; 28.5-87.5)$ & $18(24.7 ; 16.1-35.8)$ & 0.0367 \\
\hline 5 & Besoin de vasopresseurs & $2(25.0 ; 6.3-62.3)$ & $2(2.7 ; 0.7-10.3)$ & $0.0467^{* *}$ \\
\hline 6 & $\mathrm{TAM} \leq 65 \mathrm{mmHg}(\geq 0 \mathrm{~min})$ & $5(62.5 ; 28.5-87.5)$ & $18(24.7 ; 16.1-35.8)$ & 0.0334 \\
\hline
\end{tabular}

Tableau 6. Incidence d'HPI (hypotension post-intubation) selon moment de survenue de la $1^{\text {ère }} \mathrm{HPI}$

\begin{tabular}{llc}
\hline Définition & $\begin{array}{l}\text { Intervalle mesure de tension } \\
\text { artérielle post-intubation }\end{array}$ & $\begin{array}{c}\text { Incidence HPI } \\
\left(\% ; I^{1} \text { 95\%) }\right.\end{array}$ \\
\hline 1 & TAS $\leq 90 \mathrm{~mm} \mathrm{Hg}: 0-5 \mathrm{~min}$ & $8 / 81(9.9 ; 5.1-19.1)$ \\
2 & TAS $\leq 90 \mathrm{~mm} \mathrm{Hg}: 6-15 \mathrm{~min}$ & $7 / 81(8.6 ; 4.3-17.5)$ \\
3 & TAS $\leq 90 \mathrm{~mm} \mathrm{Hg}: 16-30 \mathrm{~min}$ & $5 / 81(6.2 ; 2.6-14.4)$ \\
4 & TAS $\leq 90 \mathrm{~mm} \mathrm{Hg}:(\geq 31 \mathrm{~min})$ & $3 / 81(3.7 ; 1.2-11.2)$ \\
\hline
\end{tabular}

d'HPI pour l'intervalle de temps 31 minutes et plus est de $3.7 \%$ (Tableau 6). L'hypotension pré-intubation est présente uniquement chez les patients dont la première HPI (TAS $\leq 90 \mathrm{~mm} \mathrm{Hg}$ ) a été notée dans les intervalles 0 à 5 minutes et 6 à 15 minutes (Tableau 7). L'incidence d'HPI dans l'intervalle 0 à 5 minutes est significativement plus élevée chez les patients présentant de l'hypotension pré-intubation que chez les patients hémodynamiquement stables durant la période pré-intubation.

\section{DISCUSSION}

Cette étude avait pour but principal d'évaluer l'impact du choix de la définition sur l'incidence d'HPI. À notre connaissance, il n'existe pas de données spécifiques à ce sujet dans la littérature actuelle. À la lumière de nos résultats, l'incidence d'HPI varie significativement en fonction de la définition utilisée. Il semble que l'incidence cumulée croît lorsque le temps de mesure de la TA après l'IET augmente. Cette nouvelle évidence est importante pour permettre de comparer les études et aussi établir une définition fonctionnelle pour la clinique et la recherche. Cette période post-intubation, dont la durée est hétérogène dans la littérature, n'est pas justifiée par les études précédentes sur le sujet.
Les données hémodynamiques de notre cohorte de patient montrent que les patients atteints d'HPI ont, durant la période pré-intubation, des tensions artérielles plus basses que les patients non atteints. Ceci pourrait déceler une vulnérabilité qui touche une certaine population de patients et qui prédispose celle-ci à présenter de l'HPI.

L'un de nos objectifs secondaire était de décrire l'incidence d'HPI en comparant deux groupes de patients et ce, selon les six définitions : chez les patients atteints d'hypotension pré-intubation et chez les patients hémodynamiquement stables avant l'intubation. Cela dit, notre étude confirme également que la présence d'hypotension avant d'effectuer une IET accroît de près de trois fois le risque de présenter une HPI. Il a été décrit que l'hypotension pré-intubation est un facteur prédictif d'HPI. ${ }^{11,12}$ Les auteurs ayant mis en évidence cette association ont mesuré respectivement la tension artérielle jusqu'à 5 minutes et jusqu'à 30 minutes après l'intubation. Nos résultats suggèrent une association similaire.

L'incidence d'HPI a aussi été calculée selon le moment de survenue de la premiere hypotension. Les cinq premières minutes suivant l'intubation semblent correspondre à la période durant laquelle les patients sont le plus à risque de présenter de l'HPI.pour la première fois. Ce résultat est en accord avec la physiopathologie proposée puisque l'effet des médicaments inducteurs ainsi que l'instauration de la ventilation à pression positive surviennent dans cet intervalle de temps.

Tel que mentionné par Heffner et al, les récentes trouvailles sur l'HPI montrent que l'étape suivante serait idéalement de développer un outil pré-IET. ${ }^{33}$ Son but serait de bien identifier les patients à risque et d'appliquer une optimisation hémodynamique ou un 


\begin{tabular}{|c|c|c|c|c|}
\hline \multirow[b]{2}{*}{ Définition } & \multirow[b]{2}{*}{$\begin{array}{l}\text { Intervalle de mesure de tension } \\
\text { artérielle post-intubation }\end{array}$} & \multicolumn{3}{|c|}{ Incidence d'HPI N (\%; IC 95\%) } \\
\hline & & $\begin{array}{l}\text { Hypotension pré-intubation } \\
\text { présente } \mathrm{N}=8^{*}\end{array}$ & $\begin{array}{c}\text { Hypotension pré-intubation } \\
\text { absente } \mathrm{N}=73\end{array}$ & $\begin{array}{c}\text { Valeur } \\
\mathrm{p}\end{array}$ \\
\hline 1 & $0-5 \mathrm{~min}$ & $4(50.0 ; 25.0-100.0)$ & $4(5.5 ; 2.1-14.2)$ & 0.0002 \\
\hline 2 & $6-15 \min$ & $1(12.5 ; 2.0-78.2)$ & $6(8.2 ; 3.8-17.7)$ & n.s $s^{\dagger}$ \\
\hline 3 & $16-30 \mathrm{~min}$ & 0 & $5(6.9 ; 3.0-15.1)^{* *}$ & $\mathrm{n} \cdot \mathrm{a}^{\ddagger}$ \\
\hline 4 & $\geq 31 \min$ & 0 & $3(4.1 ; 1.4-10.4)^{* *}$ & n.a \\
\hline
\end{tabular}

support vasopresseur pendant l'IET afin de devancer l'hypotension et de déterminer son effet sur la mortalité. Une autre possibilité d'avancement dans le domaine de l'HPI serait la réalisation d'un essai clinique utilisant un support vasopresseur pour prévenir l'HPI et déterminer l'effet de cette intervention sur la mortalité. ${ }^{13}$ Or, il n'est à ce jour pas clairement démontré que l'HPI à l'urgence soit un facteur prédictif de la mortalité intra-hospitalière. Si cette association entre l'HPI et la mortalité existe, elle pourrait être plus ou moins importante en fonction du temps de survenue de l'hypotension après l'intubation.

Notre étude représente la première étape dans notre projet d'identification d'une définition unique pour l'HPI. L'étape subséquente explorera les conséquences de l'HPI à différents temps après l'intubation et permettra peut-être l'homogénéisation de sa définition. Nous nous questionnons plus précisément sur la nature des paramètres d'HPI qui conduisent à un impact sur la mortalité intra-hospitalière. À notre avis, il est primordial d'arriver tout d'abord à un consensus au niveau de la définition de l'HPI et à une meilleure appréciation de ses conséquences afin de proposer un outil clinique à visée préventive. Nous croyons que la définition à utiliser est celle reliée à une mortalité augmentée et une plus grande taille d'échantillon est nécessaire afin de pouvoir conclure à une telle association.

L'une des forces de notre étude était le recueil standardisé ainsi qu'automatisé des données des patients à l'aide d'un système électronique de support de décision clinique (SEDC). Une supériorité impressionnante au niveau de la capture de données via la plate-forme ReaScribe + a déjà été montrée par une étude subventionnée par le FRQ-S. En effet, la comparaison entre les dossiers version papier et le dossier électronique Reascribe + a montré que le SEDC réduisait le temps nécessaire afin de compléter le dossier de 15 à $25 \%$ et augmentait le temps pour les soins au chevet du patient. De plus, $100 \%$ des données étaient disponibles via le SEDC alors que seulement $80 \%$ des données étaient disponibles via le dossier infirmier version papier. Les signes vitaux étaient recueillis automatiquement par le système informationnel à une fréquence de 2 minutes, ce qui est avantageux pour la détection d'HPI et de son moment de survenue.

\section{LIMITATIONS}

Nous sommes conscients que notre étude comprend certaines limitations telles que l'inclusion d'un seul centre académique tertiaire en traumatologie. En effet, la population étudiée n'est pas nécessairement représentative de la clientèle retrouvée dans la majorité des départements d'urgence de par la vocation traumatologique de l'Hôpital de l'Enfant-Jésus. En effet, 29.6\% des patients ont été intubés dans un contexte de traumatisme, ce qui est une proportion très élevée.

Près du quart des patients pour lesquels le code 'intubation' a été enregistré sur notre plateforme ont été exclus pour des raisons techniques et informatiques dans l'acquisition des données automatisées via cet outil qui était alors en implantation. Cet élément correspond probablement à la plus grande limitation de notre étude, car il est difficile de savoir si des épisodes d'hypotension ont été manqués chez ces patients. Cependant, une TAS inférieure à $90 \mathrm{mmHg}$ (pré intubation ou post intubation) a été identifiée chez seulement 4 des 30 patients exclus (Annexe 2). Ces données nous 
portent à croire que ces erreurs d'enregistrement ne sont pas survenues chez ces patients en raison d'une instabilité hémodynamique.

Il est à noter que l'étomidate fut un agent utilisé chez plus de la moitié des patients (62.1\%), l'explication la plus probable étant ses effets présumés minimes sur l'hémodynamie, contrairement à d'autres agents inducteurs.

De plus, le grand nombre de patients déjà intubés ayant été exclus de l'étude s'explique par le nombre important de transferts de patients traumatisés vers notre centre tertiaire de traumatologie. Une proportion de $3.9 \%$ des patients étaient triés $\mathrm{P} 3$ ou P4. Cela s'explique par des patients dont la condition était initialement moins urgente et qui ont été victimes d'une détérioration ayant nécessité une réévaluation et, dans leur cas, une IET.

Finalement, la durée du temps passé en réanimation était très variable d'un patient à l'autre. Les données n'étaient pas disponibles jusqu'à 60 minutes pour tous les patients en raison de cette variabilité. Cette limitation technique a pu influencer notre incidence d'HPI totale à la baisse ainsi que sous-estimer les écarts d'incidence entre les différentes définitions d'HPI.

\section{CONCLUSION}

L'HPI est une complication fréquente au département d'urgence. Son incidence varie de façon considérable d'une étude à l'autre. L'utilisation d'une définition avec un temps de mesure de TA plutôt qu'une autre semble avoir un impact sur l'incidence de l'hypotension post-intubation. Il est possible que l'hétérogénéité des définitions de l'HPI explique partiellement la variation observée de l'incidence dans la littérature. À notre avis, il est nécessaire d'arriver à un consensus sur la définition d'HPI afin d'en explorer les impacts potentiels et d'éventuellement, créer des outils cliniques permettant de mieux en prédire la survenue.

Remerciements: Les auteurs remercient Xavier Neveu pour son aide statistique dans l'élaboration de ce projet.

Intérêts opposés : Rien à déclarer.

\section{SUPPLEMENTARY MATERIAL}

To view supplementary material for this article, please visit http://dx.doi.org/10.1017/cem.2016.354

\section{RÉFÉRENCES}

1. Kharasch M, Graff J. Emergency management of the airway. Crit Care Clin 1995;11(1):53-66.

2. Mort TC. Anesthesia practice in the emergency department: overview, with a focus on airway management. Curr Opin Anaesthesiol 2007;20(4):373-8.

3. Shuster M, Nolan J, Barnes TA. Airway and ventilation management. Cardiol Clin 2002;20(1):23-35.

4. Manthous CA. Avoiding circulatory complications during endotracheal intubation and initiation of positive pressure ventilation. 7 Emerg Med 2010;38(5):622-31.

5. American College of Emergency Physicians. Rapidsequence intubation. Ann Emerg Med 1997;29(4):573.

6. Fasting S, Gisvold SE. Serious intraoperative problems-a five-year review of 83,844 anesthetics. Can 7 Anaesth 2002;49(6):545-53.

7. Jaber S, Amraoui J, Lefrant JY, Arich C, Cohendy R, Landreau L, et al. Clinical practice and risk factors for immediate complications of endotracheal intubation in the intensive care unit: a prospective, multiple-center study. Crit Care Med 2006;34(9):2355-61.

8. Reid C, Chan L, Tweeddale M. The who, where, and what of rapid sequence intubation: prospective observational study of emergency RSI outside the operating theatre. Emerg Med 7 2004;21(3):296-301.

9. Mort TC. Complications of emergency tracheal intubation: hemodynamic alterations-part I. 7 Intensive Care Med 2007;22(3):157-65.

10. Mort TC. Complications of emergency tracheal intubation: immediate airway-related consequences: part II. 7 Intensive Care Med 2007;22(4):208-15.

11. Lin CC, Chen KF, Shih CP, Seak CJ, Hsu KH. The prognostic factors of hypotension after rapid sequence intubation. American 7 Emerg Med 2008;26(8):845-51.

12. Green RS, Edwards J, Sabri E, Fergusson D. Evaluation of the incidence, risk factors, and impact on patient outcomes of postintubation hemodynamic instability. CFEM 2012; 14(2):74-82.

13. Heffner AC, Swords D, Kline JA, Jones AE. The frequency and significance of postintubation hypotension during emergency airway management. 7 Crit Care 2012;27(4): 417 e9-13.

14. Zed PJ, Abu-Laban RB, Harrison DW. Intubating conditions and hemodynamic effects of etomidate for rapid sequence intubation in the emergency department: an observational cohort study. Acad Emerg Med 2006;13(4): 378-83.

15. Smith DC, Bergen JM, Smithline H, Kirschner R. A trial of etomidate for rapid sequence intubation in the emergency department. 7 Emerg Med 2000;18(1):13-6.

16. Dufour DG, Larose DL, Clement SC. Rapid sequence intubation in the emergency department. $f$ Emerg Med 1995;13(5):705-10.

17. Hasegawa K, Shigemitsu K, Hagiwara Y, Chiba T, Watase H, Brown CA 3rd, et al. Association Between Repeated Intubation Attempts and Adverse Events in Emergency Departments: An Analysis of a Multicenter Prospective Observational Study. Ann Emerg Med 2012;60(6):749-54.e2. 
18. Jones AE, Yiannibas V, Johnson C, Kline JA. Emergency department hypotension predicts sudden unexpected inhospital mortality: a prospective cohort study. Chest 2006; 130(4):941-6.

19. Lalezarzadeh F, Wisniewski P, Huynh K, Loza M, Gnanadev D. Evaluation of prehospital and emergency department systolic blood pressure as a predictor of in-hospital mortality. Am Surg 2009 Oct;75(10):1009-14.

20. Schreiber MA, Aoki N, Scott BG, Beck JR. Determinants of mortality in patients with severe blunt head injury. Arch Surg 2002;137(3):285-90.

21. Sakles JC, Laurin EG, Rantapaa AA, Panacek EA. Airway management in the emergency department: a one-year study of 610 tracheal intubations. Ann Emerg Med 1998;31(3):325-32.

22. Tayal VS, Riggs RW, Marx JA, Tomaszewski CA, Schneider RE. Rapid-sequence intubation at an emergency medicine residency: success rate and adverse events during a two-year period. Acad Emerg Med 1999;6(1):31-7.

23. Griesdale DE, Bosma TL, Kurth T, Isac G, Chittock DR. Complications of endotracheal intubation in the critically ill. Intensive Care Med 2008;34(10):1835-42.

24. Mort TC. Emergency tracheal intubation: complications associated with repeated laryngoscopic attempts. Anesth Analg 2004;99(2):607-13.

25. Walls RM, Brown CA 3rd, Bair AE, Pallin DJ. Emergency airway management: a multi-center report of 8937 emergency department intubations. 7 Emerg Med 2011;41(4): 347-54.

26. Jaber S, Jung B, Corne P, Sebbane M, Muller L, Chanques G, et al. An intervention to decrease complications related to endotracheal intubation in the intensive care unit: a prospective, multiple-center study. Intensive Care Med 2010;36(2): 248-55.

27. Leibowitz AB. Tracheal intubation in the intensive care unit: extremely hazardous even in the best of hands. Crit Care Med 2006;34(9):2497-8.

28. Tam AY, Lau FL. A prospective study of tracheal intubation in an emergency department in Hong Kong. Eur 7 Emerg Med 2001;8(4):305-10.

29. Theodosiou CA, Loeffler RE, Oglesby AJ, McKeown DW, Ray DC. Rapid sequence induction of anaesthesia in elderly patients in the emergency department. Resuscitation 2011; 82(7):881-5.

30. Wong E, Fong YT, Ho KK. Emergency airway management-experience of a tertiary hospital in SouthEast Asia. Resuscitation 2004;61(3):349-55.

31. Hasegawa K, Hagiwara Y, Chiba T, Watase H, Walls RM, Brown DF, et al. Emergency airway management in Japan: Interim analysis of a multi-center prospective observational study. Resuscitation 2012;83(4):428-33.

32. Franklin C, Samuel J, Hu TC. Life-threatening hypotension associated with emergency intubation and the initiation of mechanical ventilation. Am 7 Emerg Med 1994;12(4):425-8.
33. Heffner AC, Swords DS, Nussbaum ML, Kline JA, Jones AE. Predictors of the complication of postintubation hypotension during emergency airway management. 7 Crit Care 2012;27(6):587-93.

34. Bullard MJ, Villa-Roel C, Guo X, Holroyd BR, Innes G, Schull MJ, et al. The role of a rapid assessment zone/pod on reducing overcrowding in emergency departments: a systematic review. Emerg Med 7 2012;29(5):372-8.

\section{ANNEXE 1}

\section{Calcul de la puissance à posteriori}

L'incidence d'HPI dans le groupe 0-5 minutes était de 0.15 et celle dans le groupe en tout temps était de 0.36 , pour une différence de proportion de $20 \%$. En fixant la puissance à $90 \%$ et un $p<0.05$, le nombre de paires minimal qui devait être comparées était de 57, et ce pour un index de corrélation de 0.40 . Or, 81 paires ont été comparées.

\section{ANNEXE 2}

\section{Patients exclus}

Les 40 patients ont été exclus le plus souvent pour des raisons techniques reliées au degré de confort variable du personnel vis-à-vis l'utilisation de la plate-forme durant la période de son implantation

$\mathrm{n}=5$ puisque $<16$ ans

$\mathrm{n}=30$ pour TAS manquante ou seulement des valeurs avant ou après intubation de TAS

Explication : Le personnel infirmier avait reçu une formation pour manœuvrer la plate-forme au préalable, mais certaines difficultés techniques ont été rencontrées lors de la navigation du logiciel. De ces 30 patients, 12 n'avaient pas de TAS post-intubation et 5 n'avaient pas de TAS du tout. Les 13 patients restants n'avaient pas de TAS pré-intubation.

$\mathrm{n}=5$ erreur d'enregistrement

Explication : Ces erreurs d'enregistrement correspondent à une création de fiche pour un patient qui avait les mêmes TAS tout le long de l'enregistrement des données. 